\title{
Biometric monitoring devices for assessing end points in clinical trials: developing an ecosystem
}

\author{
Stephen P. Arnerić, Jesse M. Cedarbaum, Sean Khozin, \\ Spyros Papapetropoulos, Derek L. Hill, Michael Ropacki, Jane Rhodes, \\ Penny A. Dacks, Lynn D. Hudson, Mark Forrest Gordon, Volker D. Kern, \\ Klaus Romero, George Vradenburg, Rhoda Au, Daniel R. Karlin, \\ Maurizio F. Facheris, Cheryl J. Fitzer-Attas, Ottavio V. Vitolo, Jian Wang, \\ Bradley M. Miller and Jeffrey A. Kaye
}

Regulatory authorities are increasingly engaged in evaluating novel ways to assess health, disease progression and treatment effects, for example, for type 2 diabetes, as highlighted in a recent news article (Nat. Rev. Drug Discov. 15, 666-667; 2016) ${ }^{1}$. Many view biometric monitoring devices (BMDs) as the path to a deeper understanding of disease processes and the status of patients ${ }^{2}$. The literature describes these devices using various terms, including digital biomarkers, digital measurement devices, technology-observed measures and biometric monitoring systems. We define a BMD as a biosensor that collects objective data on a biological recognition element (for example, blood glucose or sodium levels), anatomical structure (for example, tumour size, infarct size or hippocampal volume) or integrated physiological parameter (for example, heart rate, blood pressure, electroencephalography, mobility, speech and sleep patterns, social engagement or speed of information processing). These BMDs utilize algorithms to transform these data (signal output) into a format that is interpretable as a specific measure or an aggregate functional outcome $^{3-8}$. Health platforms using biometric monitoring allow efficient collection of real-time high-frequency data with decreased in-clinic assessment time, and dramatically reduce sample size to see population effects?.

BMDs can measure minimally observable changes in characteristics of patients to a higher level of resolution than possible with clinical observation. It is crucial to identify these earliest reliable measures of disease if we are to meet the goal of preventing or minimizing the impact of conditions on the lives of patients. For example, clinical trials in neurodegenerative diseases are becoming increasingly focused on earlier stages of disease to identify individuals who are genetically or otherwise susceptible prior to them developing symptoms. These studies aim to identify the earliest signs in the presymptomatic stage, well before the disease has caused major neurodegenerative damage. Disease is characterized both by symptoms - phenomena that patients report (patient-reported outcome measures (PROs) ) - and signs, which are observable measures that are often recorded by evaluator-related means and observerreported outcomes, or directly measured via performance outcomes (FIG. 1).

The ability of these devices to continuously, remotely and relatively unobtrusively record physical signs increases statistical power, enhancing both the sensitivity and specificity of change detection in those monitored ${ }^{9}$. By observing personal health and behaviour signs frequently (potentially continuously), reliable assessment of change is more likely. As digital drug development tools (dDDTs), BMDs may objectively augment PROs, and be integrated into the diagnosis, prognosis and treatment of patients. BMDs may identify evolving patterns of perceived normal function, reflecting a harbinger of an ensuing chronic disease state. Furthermore, the ability to measure early signs may enable more precise identification of phenotypic variants of a disease and allow better stratification of relevant subpopulations. In some cases, realtime data acquisition with BMDs may even enable statistical comparisons within an individual $^{9}$, supporting the practice of personalized medicine. In the long-term, the clinically relevant and actionable data created by BMDs may provide a more comprehensive view of the interrelated biochemical, structural, physiological and behavioural dynamics that occur during health, disease and treatment.

Unfortunately, a lack of clarity remains regarding the appropriate use of BMDs as tools to support drug registration trials. This gap was recently underscored by the newly appointed commissioner of the US FDA, Scott Gottlieb, who announced that the FDA will pilot a new approach towards regulation of digital health tools later this year (see Further information). This commentary provides a high-level roadmap, agnostic to therapeutic area, of key considerations to advance BMDs as objective assessments of clinically relevant end points for capturing the patient's experience in clinical trials, and in real-world observational studies. These considerations were developed by the member organizations of the Coalition Against Major Diseases (CAMD; see Further information). This consortium, founded in 2008, is one of 14 public-private partnerships of the Critical Path Institute, and focuses on creating new drug development tools and methods that advance regulatory science to accelerate the delivery of innovative treatments for various stages of Alzheimer disease, and neurodegenerative diseases with related cognitive and functional impairments.

\section{Key considerations for BMDs}

Defining the concept of interest and the context of use. Clear interpretation of data requires careful pre-specified definitions of the information assessed. Therefore, a priority to advance this rapidly evolving field is to methodically define the concept of interest (COI) that a given BMD would measure. In turn, the factors involved in developing and validating a COI will help frame an appropriate context of use (COU) that will be required for drug development clinical trials. Considerations for the conceptual foundations for qualification of clinical outcome assessments by the FDA, and the development of COIs and COUs, have been recently communicated ${ }^{10}$. Thus, before a BMD can be evaluated for use in a registration study as a $\mathrm{dDDT}$, a rigorous path of validation must be followed (TABLE 1).

Data standards. One of the opportunities in efficient scaling of BMDs for clinical research is development of data standards. Currently, there is no interoperable uniformity for BMD-derived data. Development of uniform data standards (for example, through adoption of Clinical Data Interchange Standards Consortium (CDISC) methods) would provide a scientifically based consensus on the way to record, structure and report data generated by BMDs. CDISC Foundational Standards provide "the basis for the complete CDISC suite of standards, supporting the clinical and non-clinical research process from protocol through data collection, data exchange, data management, data analysis and reporting" (TABLE 1). These standards focus on the core principles for defining research data standards and generally represent interest 


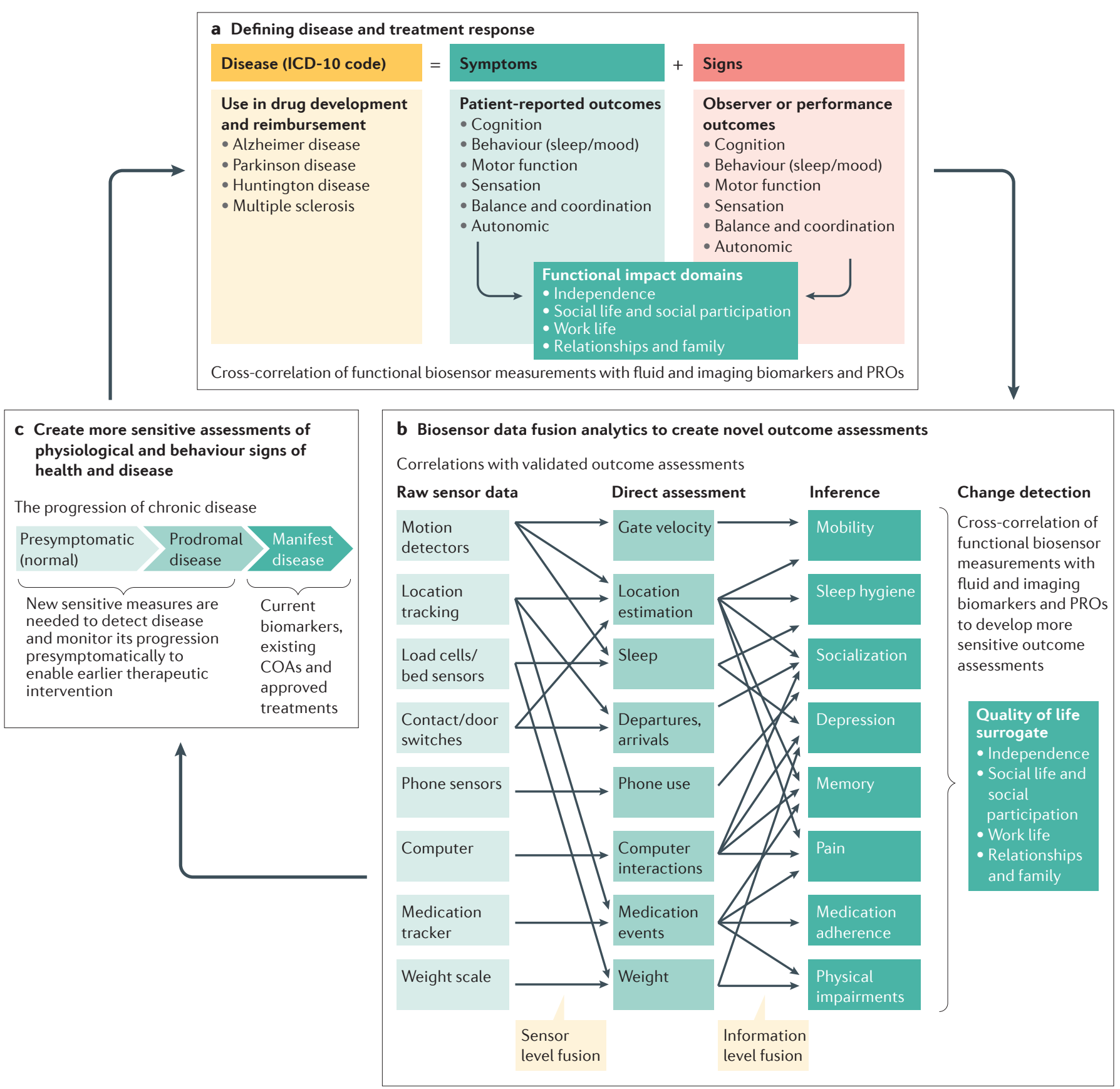

Figure 1 | Biometric monitoring devices to understand health, disease progression and treatment outcomes. a | Defining disease requires a composite understanding of both the signs (observable to the patient and others) and symptoms (reported by the patient). While these quantifiable events are not identical, they are physiologically linked (for example, increased joint pain results in a reduction in overall activity and mobility and diminished sleep quality). So, understanding how different functional domains are altered by disease and treatment is crucial in defining how an individual feels, functions and survives. $\mathbf{b}$ | Biometric monitoring devices (BMDs) can link quantifiable data collected from biosensors that measure a biological response using wearables, smart phones, clothing, implants ingestible sensors or remote biosensors (see 'Future market trends for wearables' in Further information). Improving our understanding of realtime changes in function in health and disease needs to be a focus (see 'Maximizing the potential of real world evidence to support health care innovation' in Further information). Although not yet formally validated as a clinical outcome assessment (COA), medication adherence has been shown to be decreased by as much as $40 \%$ in those with mild cognitive impairment. c | For many chronic diseases, the clinical instruments used to quantify disease are validated only during manifest disease, and there is a strong need to begin treatment earlier in the disease course, as indicated in the figure, which could be enabled by BMDs. Claims regarding the benefits of BMDs should include the analytical and clinimetric validity of the devices and the clinical utility of the measured end points (see "What the Fitbit lawsuit means for clinical researchers' in Further information). With the increased emphasis on prevention therapies, the need to identify sensitive outcome assessments is growing. Otherwise, long ( $5+$ year) trials will be necessary before the effects of early interventions can be determined. Both the health care community and regulators are embracing this need, and analysis of BMD data is anticipated to provide patient-centred, real-world evidence to support regulatory and clinical decision-making (see 'Use of real-world evidence to support regulatory decision-making for medical devices: draft guidance for industry and Food and Drug Administration staff' in Further information). PRO, patient-reported outcome. 
Table 1 | Key steps and considerations to advance biometric monitoring devices as clinical outcome assessments

\begin{tabular}{|c|c|c|}
\hline Key steps & Key considerations & Further information \\
\hline $\begin{array}{l}\text { Define } \mathrm{COI} \text { and } \\
\text { COU }\end{array}$ & $\begin{array}{l}\text { - Each COI must adhere to a series of steps that include: content validation, } \\
\text { construct validation, criterion validation, analytical validation and ultimately } \\
\text { clinical validation } \\
\text { - To lead to meaningful regulatory endorsement of BMDs as digital drug } \\
\text { development tools, clear COU statements must be developed. Each COU is } \\
\text { based on the current state of evidence and a clear rationale for its application } \\
\text { in the drug development process, or eventually, in health care management } \\
\text { - Categories of biomarker currently detailed by the FDA-NIH include: } \\
\text { susceptibility/risk biomarker; diagnostic biomarker; monitoring biomarker; } \\
\text { prognostic biomarker; predictive biomarker; pharmacodynamics/response } \\
\text { biomarker and safety biomarker. An end point being evaluated for its ability to } \\
\text { predict clinical benefit would be a candidate surrogate end point } \\
\text { - As the impact of the clinical decision for the patient grows, the level of } \\
\text { evidence needed to support that COU will increase }\end{array}$ & $\begin{array}{l}\text { - Clinical Outcome Assessment Qualification } \\
\text { Program: http://www.fda.gov/Drugs/ } \\
\text { DevelopmentApprovalProcess/ } \\
\text { DrugDevelopmentToolsQualificationProgram/ } \\
\text { ucm284077.htm } \\
\text { BEST resource: } \text { https://www.ncbi.nlm.nih.gov/ } \\
\text { books/NBK326791/ }\end{array}$ \\
\hline $\begin{array}{l}\text { Defining data } \\
\text { standards for } \\
\text { actionable } \\
\text { databases }\end{array}$ & $\begin{array}{l}\text { - Data standards can enable prospective collection of data in a standardized } \\
\text { format in both clinical trials and observational studies, allow integration of } \\
\text { various data sources to quantify the predictive accuracy, utility and reliability } \\
\text { of BMDs and expedite submissions and reviews to regulatory authorities } \\
\text { - In } 2014 \text {, the FDA issued a guidance document on 'Providing Regulatory } \\
\text { Submissions In Electronic Format - Standardized Study Data'. Technical } \\
\text { specifications associated with this guidance are updated periodically }\end{array}$ & $\begin{array}{l}\text { - Clinical Data Interchange Standards } \\
\text { Consortium foundational standards: } \\
\text { https://www.cdisc.org/standards/ } \\
\text { foundational-standards } \\
\text { - FDA guidance on electronic data submissions: } \\
\text { https://www.fda.gov/downloads/drugs/ } \\
\text { guidances/ucm384686.pdf }\end{array}$ \\
\hline $\begin{array}{l}\text { Assurance of } \\
\text { GCP standards }\end{array}$ & $\begin{array}{l}\text { - The intended subject for data collection must be verified. Passwords or dual } \\
\text { factor authentication are normally used } \\
\text { - A complete audit trail from device to the clinical trial database must be } \\
\text { established } \\
\text { - Well-defined procedures are needed to confidently identify artifacts in data } \\
\text { - BMDs often operate in a complex and heterogeneous computer system } \\
\text { environment. For example, a watch measuring activity might itself run software } \\
\text { and communicate with a smartphone application, a cloud server and a clinical } \\
\text { trial database. Software environments may change (including automatic } \\
\text { upgrades). Protocols to distinguish changes in BMD measurements due to } \\
\text { patient changes versus software environment changes are essential } \\
\text { - The requirements outlined in FDA } 21 \text { CFR part } 11 \text { are an important } \\
\text { consideration for electronic submission of BMD data to the FDA } \\
\text { - Patient privacy must be protected }\end{array}$ & $\begin{array}{l}\text { - FDA GCP guidance: https://www.fda.gov/ } \\
\text { scienceresearch/specialtopics/runningclini- } \\
\text { caltrials/guidancesinformationsheetsandno- } \\
\text { tices/ucm219433.htm }\end{array}$ \\
\hline
\end{tabular}

BEST, Biomarkers, EndpointS, and other Tools; BMD, biometric monitoring device; COI, concept of interest; COU, context of use; GCP, good clinical practice.

areas that are common across all studies such as demographics, medical history, medication history and concomitant medications, adverse events and other common domains.

The standardization of how data are collected, stored, labelled and tagged allows for the exchange of information, facilitates the pooling of data from different devices, and allows contextualization of the data. For example, recording the timing of data collection of blood glucose readings is critical metadata for understanding whether the measurement occurs after fasting or immediately after a meal. Similarly, when sharing data from multiple actigraphy devices, labelling and tagging data with instrument properties and pre-processing will support data pooling. Providing contextualization and standardization will reduce dependence on black-box and proprietary algorithms, making data comparable at all stages of processing. This would enable processing steps themselves to be treated as a form of data and tracked in the same standardized ontologies in which the data and metadata themselves belong.

Good clinical practice. Within the pharmaceutical industry, the use of BMDs for exploratory work or internal decisionmaking is becoming a more routine practice. Development of Good Clinical Practice (GCP) standards will be crucial to enable the ethical and correct use of BMD-derived data from later-stage clinical trials for regulatory submission (TABLE 1).

Creating a global ecosystem. Creation of a unified, global, precompetitive BMD ecosystem including both the medical device and drug development spaces would advance the necessary conversations and provide a network of experts to implement BMD use in future routine health care management (TABLE 1). These experts would include clinicians, data scientists and managers, psychometricians, pharmacometricians, pharmacoeconomists, regulators and statisticians from the device and pharmaceutical industries, regulatory agencies, academia and government. Accomplishing this objective would augment the ongoing US Precision Medicine Initiative, and could provide an evidence-based path to integrate both clinical drug trial and real-world data.

A unified lexicon of precise terminologies for the potential multitude of BMDs is needed to facilitate dialogue in this community. The glossary developed by the FDA-NIH working group (BEST - Biomarkers, EndpointS, and other Tools - Resource; see Further information) can serve as an important foundation to develop this lexicon. 
Standardized data sharing would increase the efficiency of BMD development and accelerate the iterative learning required to support outcome assessments for a particular COU. Multiple organizations are pursuing the advancement of BMDs in parallel, but a lack of appropriate incentives, coupled with insufficient allocation of resources, have created substantial barriers to sharing and assembling patient-level data. One step to help address this could be the establishment of a datasharing consortium to generate consensus on data standards to accelerate the use of BMDs in clinical drug development.

Providing consensus guidelines for what constitutes sufficient or necessary validation of BMDs depends on the COU and the related health-care decisions. However, if BMDs are used in future registration studies, BMDs should undergo the same psychometric validation process as other clinical outcomes assessments (such as PROs) to be considered reliable and valid measures. Real-time data acquisition with BMDs can enable statistical comparisons within an individual, supporting the practice of personalized medicine. Integration of existing standards with novel methods will require rigorous cross-validation and testing by the medical community. Active dialogue with the regulators is imperative before this work is initiated, and must happen periodically, at important decision points.

Overall, by involving multiple stakeholders in precompetitive, public-privatepartnerships, the community could align on principles and advance a clear roadmap to develop, validate and standardize the use of BMDs in various diseases.

Stephen P. Arnerič, Lynn D. Hudson, Volker D. Kern and Klaus Romero are at the Critical Path Institute, Tucson, Arizona, USA.
Jesse M. Cedarbaum and Jane Rhodes are at Biogen, Cambridge, Massachusetts, USA

Sean Khozin is at the Food and Drug Administration, Bethesda, Maryland, USA.

Spyros Papapetropoulos was previously at TEVA, Cambridge, Massachusetts, USA. Present address: Massachusetts General Hospital, Harvard, Boston, Massachusetts, USA.

Derek L. Hill is at IXICO, London, UK.

Michael Ropacki is at Loma Linda University, Loma Linda, California, USA.

Penny A. Dacks was previously at at the Alzheimer's Drug Discovery Foundation, New York, New York, USA. Present address: American Epilepsy Association, Chicago, Illinois, USA.

Mark Forrest Gordon was previously at Boehringer Ingelheim Pharmaceuticals, Ridgefield, Connecticut, USA. Present address: TEVA, Malvern, Pennsylvania, USA.

George Vradenburg is at UsAgainstAlzheimer's, Washington, DC, USA.

Rhoda Au is at Boston University, Boston, Massachusetts, USA.

Daniel R. Karlin is at Pfizer, Boston, Massachusetts, USA. Ottavio V. Vitolo was previously at Pfizer, Boston, Massachusetts, USA. Present address: Homology Medicines, Boston, Massachusetts, USA.

Maurizio F. Facheris is at AbbVie, North Chicago, Illinois, USA.

Cheryl J. Fitzer-Attas is at CHDI Foundation, Princeton, New Jersey, USA.

Jian Wang and Bradley M. Miller are at Eli Lilly \& Company, Indianapolis, Indianapolis, USA.

Jeffrey A. Kaye is at Oregon Health \& Science University, Portland, Oregon, USA.

Correspondence to S.A. sarneric@c-path.org doi: $10.1038 /$ nrd. 2017.153 Published online 22 Sep 2017

1. Kingwell, K. FDA eyes new diabetes end points. Nat. Rev. Drug Discov. 15, 666-667 (2016).

Delude, C. M. Deep phenotyping: The details of disease. Nature 527, S14-S15 (2015).

3. Espay, A. J. et al. Technology in Parkinson's disease: challenges and opportunities: technology in PD. Mov. Disord. (2016). doi: 10.1002/mds. 26642

4. Kaye, J. et al. Unobtrusive measurement of daily computer use to detect mild cognitive impairment. Alzheimers Dement. 10, 10-17 (2014).
5. Dodge, H. H., Mattek, N. C., Austin, D., Hayes, T. L. \& Kaye, J. A. In-home walking speeds and variability trajectories associated with mild cognitive impairment. Neurology 78, 1946-1952 (2012).

6. Austin, D., Cross, R. M., Hayes, T. \& Kaye, J. Regularity and predictability of human mobility in personal space. PLOS ONE 9, e90256 (2014).

7. Dodge, H. H., Zhu, J., Lee, C.-W., Chang, C.-C. H. \& Ganguli, M. Cohort effects in age-associated cognitive trajectories. J. Gerontol. A. Biol. Sci. Med. Sci. 69, 687-694 (2014).

8. Hayes, T. L., Riley, T., Mattek, N., Pavel, M. \& Kaye, J. A. Sleep habits in mild cognitive impairment. Alzheimer Dis. Assoc. Disord. 28, 145-150 (2014).

9. Dodge, H. H. et al. Use of high-frequency in-home monitoring data may reduce sample sizes needed in clinical trials. PLOS ONE 10, e0138095 (2015).

10. Walton, M. K. et al.. Clinical outcome assessments: conceptual foundation-report of the ISPOR Clinical outcomes assessment - emerging good practices for outcomes research task force. Value Health 18 , 741-752 (2015).

\section{Acknowledgements}

This work was supported, in part, by grant number 1 U18FD005320 from the U.S. Food and Drug Administration's Critical Path Public Private Partnerships Grant, and members including: AbbVie, Alzheimer's Association, Alzheimer's Disease Discovery Foundation (ADDF), Alzheimer's Research UK, AstraZeneca, Biogen, Boehringer Ingelheim, CHDI Foundation, Lilly, Merck, Novartis, Pfizer, and UsAgainstAlzheimer's.

\section{Competing interests statement}

The authors declare no competing interests.

\section{FURTHER INFORMATION}

Fostering Medical Innovation: A Plan for Digital Health Devices: https://blogs.fda.gov/fdavoice/index.php/2017/06/ fostering-medical-innovation-a-plan-for-digital-health-devices Coalition Against Major Diseases (CAMD) website: https://c-path.org/programs/camd/ Biomarkers, EndpointS, and other Tools (BEST) resource: https://www.ncbi.nlm.nih.gov/books/NBK326791/

Future market trends for wearables: https://www. meddeviceonline.com/doc/future-market-trends-forwearable-devices-0001

Maximizing the potential of real world evidence to support health care innovation: http://www.nehi.net/writable/

publication files/file/nehi 2016 rwe statement.pdf

What the Fitbit lawsuit means for clinical researchers: https://techcrunch.com/2016/08/18/what-the-fitbit-lawsuitmeans-for-clinical-researchers

Use of real-world evidence to support regulatory decisionmaking for medical devices: draft guidance for industry and Food and Drug Administration staff: https://www.fda.gov/ downloads/medicaldevices/deviceregulationandguidance/ guidancedocuments/ucm513027.pdf

ALL LINKS ARE ACTIVE IN THE ONLINE PDF 